Adriana Orcesi Pedro ${ }^{1}$

Aarão Mendes Pinto Neto 1

Lucia Helena Simões da Costa Paiva 1

Maria José Osis 1

Ellen Hardy 1

\title{
Idade de ocorrência da menopausa natural em mulheres brasileiras: resultados de um inquérito populacional domiciliar
}

\author{
Age at natural menopause among Brazilian \\ women: results from a population-based survey
}

1 Departamento de Tocoginecologia Faculdade de Ciências Médicas, Universidade Estadual de Campinas. Rua Alexander Fleming 101, Campinas, $S P$ 13083-970, Brasil.

\begin{abstract}
A descriptive, exploratory, cross-sectional, population-based study was conducted to identify age at menopause and its associated factors as well as socio-cultural, demographic, and economic characteristics of climacteric women in Campinas, São Paulo State. Subjects were 456 Brazilian women between 45 to 60 years of age, selected through area cluster sampling. Data were collected through home interviews using a structured, pre-tested questionnaire. This instrument was an adaptation, done by the authors, of questionnaires provided by the International Health Foundation/International Menopause Society and by the North American Menopause Society. Statistical analysis was performed through life table analysis and Cox logistic regression. Mean age at menopause was 51.2 years. There was no association between study factors and age at natural menopause.
\end{abstract}

Key words Menopause; Cross-Sectional Studies; Women's Health

Resumo Realizou-se um estudo descritivo e exploratório de corte transversal, de base populacional, com o objetivo de estudar a idade à menopausa e seus fatores associados e características sócio-culturais, demográficas e econômicas em mulheres climatéricas, residentes no Município de Campinas, Estado de São Paulo. Selecionaram-se, através de amostragem por conglomerados, 456 mulheres, na faixa etária de 45-60 anos de idade. Os dados foram coletados através de entrevistas domiciliares, com um questionário estruturado e pré-testado. O questionário foi o resultado da adaptação, feita pelos autores, de questionários fornecidos pela Fundação Internacional de Saúde/Sociedade Internacional de Menopausa e pela Sociedade Norte-Americana de Menopausa. Para análise dos dados, foi utilizada a curva de sobrevivência pelo método de Tabela de Vida para estimar a taxa acumulada de mulheres na menopausa natural, e para determinar os fatores associados à idade da menopausa foi utilizada a análise de regressão logística de Cox. A média etária da ocorrência da menopausa natural foi de 51,2 anos. Não houve associação entre os fatores estudados com a idade à menopausa natural.

Palavras-chave Menopausa; Estudos Transversais; Saúde da Mulher 


\section{Introdução}

A análise dos fatores associados à idade de ocorrência da menopausa tem diversas implicações clínicas e epidemiológicas. A menopausa tardia tem sido associada com baixo risco de osteoporose, de doenças cardiovasculares e alta freqüência de câncer de mama, ovário e endométrio. Além disso, pacientes na faixa etária dos 40 anos, freqüentemente querem saber quando se tornarão menopausadas, que características podem predispor a uma menopausa mais precoce, e se as circunstâncias ou estilo de vida fazem alguma diferença nesse período.

Recentemente, vários relatos têm mostrado que o tabagismo, baixo nível sócio-econômico e baixo peso ponderal estão associados à menopausa precoce. Os fatores que reduzem os ciclos ovulatórios durante o período reprodutivo, como a paridade, uso de contraceptivos hormonais e ciclos anovulatórios têm tendência a postergar a idade em que ocorre a última menstruação. Portanto, o conhecimento dos fatores relacionados à idade à menopausa pode fornecer dados para interpretação dessas associações e pode, em princípio, sugerir medidas preventivas. Além disso, os potenciais fatores determinantes da idade à menopausa podem diferir em populações com características diferentes que influenciam a idade de ocorrência (Parazzini et al., 1992).

Não há estudos epidemiológicos de base populacional realizados em mulheres brasileiras, sendo que os estudos nacionais a respeito da idade à menopausa foram calculados apenas em mulheres que freqüentam serviços médicos e, portanto, considerados não representativos da população geral. Existem poucos dados sobre essas questões em populações latinoamericanas, que diferem em estilo de vida e hábitos reprodutivos de populações de países desenvolvidos. Acredita-se que conhecendo melhor a média etária na menopausa e as características sociodemográficas e reprodutivas de mulheres climatéricas, os serviços e profissionais de saúde terão condições de estar melhor capacitados para responder às necessidades dessas mulheres, quer através da orientação adequada, quer através do apoio psicológico, quer através das alternativas diagnósticas e terapêuticas indicadas em diferentes situações.

Dessa forma, os objetivos deste estudo foram conhecer as principais características sociodemográficas de mulheres climatéricas entre 45 a 60 anos de idade, residentes no Município de Campinas, conhecer a média etária de ocorrência da menopausa natural com seus fa- tores associados e a proporção de mulheres na pós-menopausa, de acordo com a idade.

\section{Sujeitos e métodos}

Tratou-se de um estudo descritivo e exploratório de corte transversal, tipo inquérito populacional domiciliar. Para o cálculo do tamanho amostral considerou-se a proporção populacional estimada de $36 \%$, formada por mulheres com idade à menopausa de até 48 anos $(\mathrm{Pa}-$ razzini et al., 1992), com uma diferença máxima desejada entre a proporção amostral e populacional de $5 \%$, e um erro tipo I (alfa) de 0,05 . O número mínimo calculado de entrevistas foi de 367 mulheres, mas foi acrescido de $20 \%$, resultando em um total de 456 entrevistas. Isso se justificou pela possibilidade de recusas ou perdas durante o trabalho de campo.

A seleção dos sujeitos foi por conglomerados, a unidade de referência foi um setor censitário, conforme definido pelo IBGE (Fundação Instituto Brasileiro de Geografia e Estatística): a menor unidade de amostragem, geralmente composta por vários quarteirões, e outras vezes por uma favela. Estudaram-se 82 setores que foram sorteados entre todos os setores censitários de Campinas, cujo número total é de 845. Em cada um dos setores selecionaram-se no mínimo uma e no máximo seis mulheres. As mulheres que participaram do estudo foram selecionadas através de entrevista domiciliar. Para fazer uma seleção aleatória das mulheres, adotou-se amostragem sistemática com intervalo de seis endereços entre as casas em que as entrevistadoras deveriam obter informações sobre as mulheres residentes (Kish, 1972).

Para as mulheres selecionadas que aceitavam participar, a entrevistadora lia o Termo de Consentimento Livre e Esclarecido, redigido conforme as normas do Conselho Nacional de Saúde, do Ministério da Saúde. Os critérios de inclusão foram ser mulher entre 45 a 60 anos de idade, residir em Campinas e ser brasileira nata. Foram excluídas aquelas que apresentaram recusa explícita ou incapacidade em participar do estudo e qualquer fator que impedisse a entrevista.

Foram estudadas as seguintes variáveis: idade, idade à menarca, idade à menopausa (idade em que ocorreu a última menstruação, seguida por um período mínimo de 12 meses de amenorréia), cor, tabagismo, índice de massa corpórea (peso/altura 2 ), estado marital, paridade, escolaridade, emprego e classe socioeconômica (Almeida \& Wikerhauser, 1991).

Um questionário estruturado e pré-testado foi usado para obter as informações relatadas 
pelas mulheres. O questionário utilizado foi elaborado a partir de outros dois e adaptado pelos autores. O primeiro questionário foi fornecido pela Sociedade Internacional de Menopausa e Fundação Internacional de Saúde, após ter sido aplicado em sete países do Sudeste Asiático em 1993 (Boulet et al., 1994). O segundo, que havia sido aplicado nos Estados Unidos, foi fornecido pela Sociedade Norte-Americana de Menopausa (Utian \& Schiff, 1994).

O trabalho de campo foi realizado por uma equipe de quatro entrevistadoras e uma supervisora, após 32 horas de treinamento.

A coleta de dados foi iniciada em outubro de 1997 e encerrada em janeiro de 1998. A proporção de recusas foi de $11,2 \%(64 / 571)$. Para cada mulher entrevistada foram visitados 3,8 domicílios residenciais $(456 / 1.771)$, sendo que na maior parte deles as mulheres residentes estavam fora da faixa etária fixada para o estudo.

Para a análise dos dados utilizou-se o pacote SPSS.

Utilizou-se a curva de sobrevivência pelo Método de Tabela de Vida para estimar a taxa acumulada de mulheres na menopausa natural de acordo com a idade. Para determinar os fatores associados à idade à menopausa, foi utilizado o teste de Wilcoxon generalizado e a análise de regressão múltipla de Cox. Os fatores que foram explorados nesta análise foram: cor, paridade, número de abortamentos, idade à menarca, índice de massa corpórea, estado marital, escolaridade, emprego, antecedente de uso de contraceptivos hormonais e não-hormonais, ligadura tubária, tabagismo e classe social.

\section{Resultados}

\section{Aspectos sócio-demográficos}

A distribuição etária foi semelhante nas três categorias de faixas etárias. A maioria das mulheres foi branca e de baixo nível educacional, sendo que aproximadamente $70 \%$ referiram escolaridade de no máximo quatro anos. A grande maioria vivia com companheiro e não possuía emprego remunerado. A religião predominante foi a católica. Aproximadamente dois terços pertenciam às classes socioeconômicas C e D. Cerca de $75 \%$ das mulheres entrevistadas estavam na peri ou pós-menopausa. A procedência das mulheres foi predominantemente da Região Sudeste $(80,9 \%)$, sendo que deste total, $64,4 \%$ procediam do Estado de São Paulo (Tabela 1). Cerca de 17,5\% sempre moraram em Campinas.

\begin{tabular}{|c|c|c|}
\hline \multicolumn{3}{|c|}{$\begin{array}{l}\text { Principais características demográficas } \\
\text { da população estudada ( } n=456 \text { ). }\end{array}$} \\
\hline Características & $\mathrm{n}$ & $\%$ \\
\hline \multicolumn{3}{|l|}{ Distribuição etária } \\
\hline $45-49$ & 172 & 37,6 \\
\hline $50-54$ & 144 & 31,6 \\
\hline $55-60$ & 140 & 30,8 \\
\hline \multicolumn{3}{|l|}{ Cor } \\
\hline Branca & 257 & 56,4 \\
\hline Negra/parda & 125 & 27,4 \\
\hline Outras & 74 & 16,2 \\
\hline \multicolumn{3}{|l|}{ Nível educacional } \\
\hline Analfabeta & 72 & 15,7 \\
\hline Primário básico (até 4 anos) & 243 & 53,7 \\
\hline Primário completo & 81 & 17,7 \\
\hline Secundário & 43 & 9,3 \\
\hline Universitário & 17 & 3,6 \\
\hline \multicolumn{3}{|l|}{ Estado marital } \\
\hline Solteira & 28 & 6,2 \\
\hline Casada/amasiada & 322 & 70,6 \\
\hline Separada/divorciada & 52 & 11,4 \\
\hline Viúva & 54 & 11,8 \\
\hline \multicolumn{3}{|l|}{ Emprego } \\
\hline Período integral & 126 & 27,6 \\
\hline Período parcial & 38 & 8,3 \\
\hline Nenhum & 292 & 64,1 \\
\hline \multicolumn{3}{|l|}{ Religião } \\
\hline Católica & 324 & 71,1 \\
\hline Evangélica & 82 & 18,0 \\
\hline Outras & 50 & 10,9 \\
\hline \multicolumn{3}{|l|}{ Classe social } \\
\hline Classes A e B & 91 & 20,0 \\
\hline Classes C e D & 323 & 70,6 \\
\hline Classe E & 42 & 9,2 \\
\hline \multicolumn{3}{|l|}{ Estado menopausal } \\
\hline Pré-menopausa & 117 & 25,8 \\
\hline Perimenopausa & 80 & 17,6 \\
\hline Pós-menopausa & 259 & 56,6 \\
\hline \multicolumn{3}{|l|}{ Procedência } \\
\hline Sudeste & 369 & 80,9 \\
\hline Sul & 48 & 10,5 \\
\hline Norte/Nordeste & 32 & 7,0 \\
\hline Centro-Oeste & 7 & 1,6 \\
\hline
\end{tabular}




\section{Idade de ocorrência da menopausa}

A idade mínima de ocorrência da menopausa natural foi de 28 anos e a máxima de 58 , sendo a média etária de 51,2 anos. Aproximadamente $60 \%$ do total de mulheres estavam na pós-menopausa com 51 anos ou menos (Figura 1). Aproximadamente $5,5 \%$ das mulheres apresentaram menopausa precoce (abaixo de 40 anos) e $2 \%$ apresentaram menopausa tardia (acima de 55 anos).

O tempo decorrido desde a menopausa até a entrevista variou de um a 30 anos, com média de 7,2 anos (DP = 5,0). Aproximadamente 70\% das mulheres tiveram tempo médio decorrido da menopausa de oito anos ou menos (Figura 2).

\section{Fatores associados à idade de ocorrência} da menopausa

Não houve associação estatisticamente significativa entre o hábito de fumar e a média etária de ocorrência da menopausa (Tabela 2).

Não houve associação entre as variáveis sociodemográficas como a cor, a escolaridade, o emprego, a classe social e o estado marital com a idade da ocorrência da menopausa (Tabela 3).

Em mulheres com antecedentes de uso de método anticoncepcional hormonal, a mediana de idade de ocorrência da menopausa foi mais alta do que as mulheres que não os utilizaram durante qualquer período da sua vida reprodutiva, sendo essa diferença estatisticamen- te significante. Mulheres com índice de massa corpórea maior que $30 \mathrm{~kg} / \mathrm{m}^{2}$ apresentaram mediana de idade da ocorrência da menopausa cerca de 1,1 anos mais alta do que as com índice de massa corpórea menor que $30 \mathrm{~kg} / \mathrm{m}^{2}$, porém sem significância estatística (Tabela 4).

Através da Análise de Regressão Logística de Cox não houve associação entre os fatores estudados e a idade de ocorrência da menopausa (Tabela 5).

\section{Discussão}

A média etária à menopausa encontrada neste estudo foi semelhante à registrada em mulheres de países industrializados do Ocidente, que é ao redor de 50 anos (McKinlay et al., 1972).

Em sociedades ocidentais, McKinlay et al. (1972) relataram uma média etária à menopausa em mulheres da Grã-Bretanha ao redor dos 50,2 anos. Na Holanda, essa média foi de 51,5 anos (Brand \& Lehert, 1978) e nos Estados Unidos, ao redor de 51,1 anos (Kato et al., 1998). Benjamin (1960), estudando mil mulheres brancas na África do Sul observou que a média etária à menopausa foi ao redor de 46,7 anos e em Gana essa média foi ao redor de 48 anos (Kwawukume et al., 1993). Chompootweep et al. (1993) relataram a média etária de ocorrência da menopausa em mulheres tailandesas ao redor de 45 anos e Boulet et al. (1994), em um estudo de corte transversal realizado em sete paí-

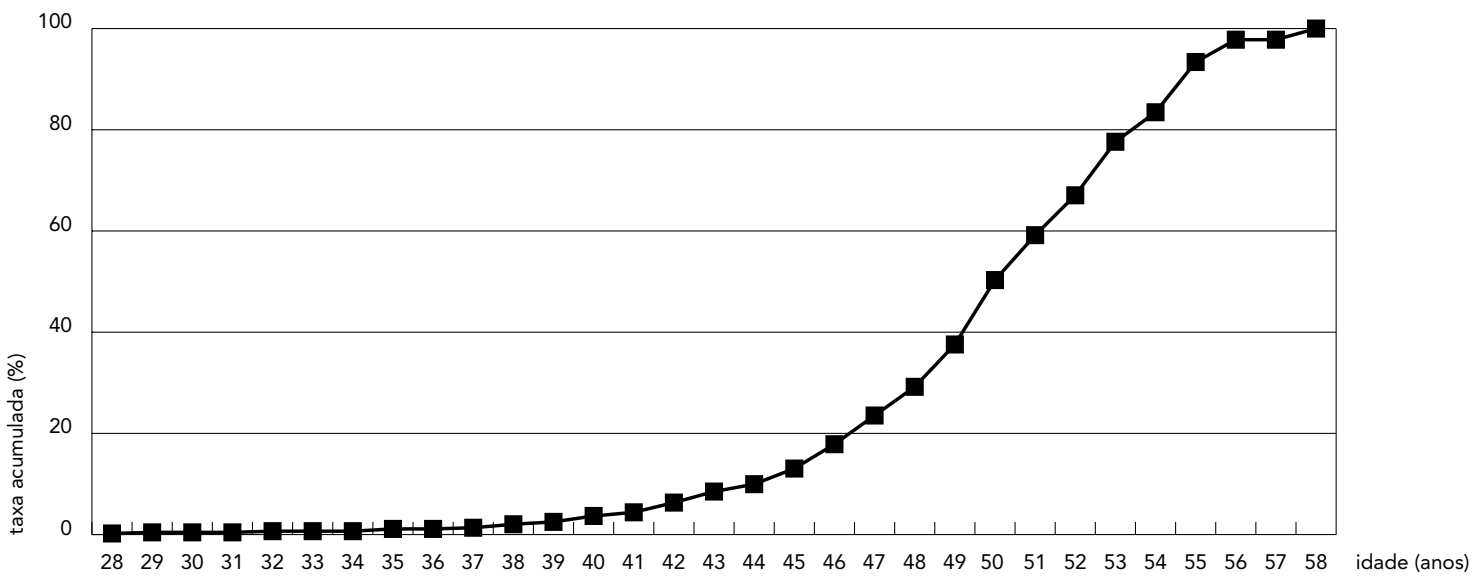

Nota: Método de tabela de vida. 


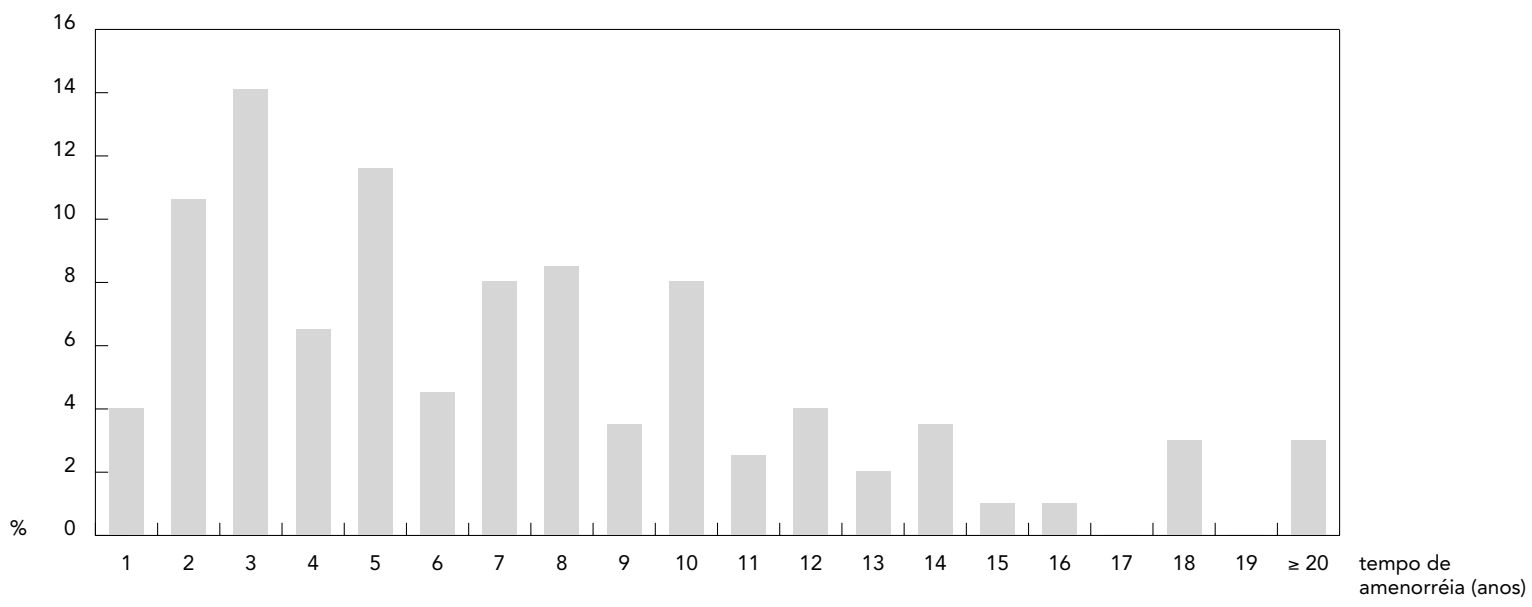

* Amostra composta apenas de mulheres na pós-menopausa e excluindo as mulheres submetidas à histerectomia e/ou ooforectomia (menopausa cirúrgica).

ses do Sudeste Asiático, revelaram que essa média era ao redor de 51,1 anos. Estudo populacional recente, realizado nos Emirados Árabes Unidos, revelou ser essa média ao redor dos 47,3 anos (Rizk et al., 1998).

No Brasil não há dados oriundos de estudos de base populacional, mas em pesquisas realizadas com população hospitalar, a média etária à menopausa foi ao redor dos 45,1 a 48,5 anos (Fonseca et al., 1985; Halbe et al., 1990; Pinto Neto et al., 1992; Wehba et al., 1998). A média etária de ocorrência da menopausa nesses estudos realizados com mulheres que freqüentam serviços de saúde, foi inferior à média encontrada neste estudo de base populacional, provavelmente porque a coleta de dados de mulheres de população hospitalar envolve patologias que possam influenciar a idade da menopausa.

As causas das diferenças entre as idades de ocorrência da menopausa natural nos diversos países não são claras. Embora seja aceito que fatores sócio-econômicos possam estar indiretamente envolvidos na idade de ocorrência da menopausa, quer através da educação, nutrição ou estado de saúde, neste estudo a idade à menopausa foi semelhante nos diversos níveis sócio-econômicos. Em geral, populações de baixo nível sócio-econômico têm maior paridade quando comparadas às populações de maior renda, e esse fato pode se contrapor aos outros fatores, já que a paridade tem sido associada a uma menopausa mais tardia (McKinlay et al., 1972).
Uma possível explicação para as diferenças relatadas nas distintas populações é que entrevistas realizadas de forma retrospectiva geralmente subestimam a idade à menopausa. A média também tende a subestimar a idade à menopausa; seria mais acurado usar a mediana; mais ainda, mesmo em estudos prospectivos não há uma concordância se a menopausa começa com o último episódio de sangramento ou após a amenorréia estar presente por um ano (Ginsburg, 1991).

Apesar destas observações, a média etária de ocorrência da menopausa não mudou muito desde os relatos de Aristóteles e Hipócrates há quase dois mil anos, onde o relato da idade da ocorrência da menopausa era por volta da quarta década. Autores medievais relataram que a parada da menstruação ocorria por volta dos 50 anos, muito próxima da média etária à menopausa da mulher do século XX, tanto nas mulheres brasileiras como nas de países desenvolvidos do Ocidente.

Mas afinal, o que determina a idade da ocorrência da menopausa? Para os vários fatores que podem influenciar essa idade, as evidências epidemiológicas são pobres. Neste estudo, contudo, não encontramos fatores sociodemográficos ou reprodutivos associados à idade à menopausa.

$\mathrm{O}$ fator mais importante para determinar a idade da ocorrência da menopausa é o número de folículos ovarianos. A célula germinativa pri- 
Tabela 2

Idade mediana à menopausa segundo características de tabagismo ( $n=455)$.

\begin{tabular}{lrrr}
\hline Variável & Idade mediana & $\mathbf{n}$ & $\mathbf{p}^{*}$ \\
\hline Hábito de fumar & & & 0,363 \\
Fuma atualmente & 50,9 & 93 & \\
Fumou no passado & 51,4 & 66 & \\
Nunca fumou & 51,4 & 296 & 0,382 \\
& & & \\
Número de cigarros/dia & & 296 & \\
0 & 51,4 & 58 & \\
$1-9$ & 51,2 & 98 & \\
$\geq 10$ & 51,0 & & \\
Tempo que fuma/fumou (anos) & & 302 & \\
0 & 51,4 & 54 & \\
$1-15$ & 50,8 & 98 & \\
$>15$ & 51,6 & & \\
\hline
\end{tabular}

* Teste de Wilcoxon generalizado (Gehan).

Tabela 3

Idade mediana à menopausa segundo características sócio-demográficas $(n=455)$.

\begin{tabular}{|c|c|c|c|}
\hline Variável & Idade mediana & $\mathbf{n}$ & $p^{*}$ \\
\hline Cor & & & 0,214 \\
\hline Branca & 51,0 & 257 & \\
\hline Parda, mulata, preta & 51,8 & 124 & \\
\hline Outra & 51,0 & 74 & \\
\hline Escolaridade & & & 0,398 \\
\hline Analfabetismo & 53,0 & 70 & \\
\hline 1ạ-4ạ série & 51,0 & 242 & \\
\hline$>4 a$ série & 51,0 & 141 & \\
\hline Emprego & & & 0,731 \\
\hline Período integral & 51,3 & 126 & \\
\hline Período parcial & 52,0 & 38 & \\
\hline Nenhum & 51,1 & 291 & \\
\hline Classe social & & & 0,183 \\
\hline Classes A e B & 51,7 & 91 & \\
\hline Classes C, D e E & 51,2 & 363 & \\
\hline Estado marital & & & 0,315 \\
\hline Sem companheiro & 51,1 & 134 & \\
\hline Com companheiro & 51,4 & 321 & \\
\hline
\end{tabular}

* Teste de Wilcoxon generalizado (Gehan). mordial separa-se da célula somática em um estágio inicial da embriogênese. Entre 1.0002.000 migram para a crista gonadal, onde elas se multiplicam rapidamente, chegando ao máximo de cinco a sete milhões de folículos ao redor do quinto mês de vida intra-uterina, quando essa multiplicação pára. A partir de então há uma perda dos folículos primordiais do ovário fetal até que, ao nascimento, cada ovário contenha cerca de um milhão de folículos. Esse número continua a diminuir após o nascimento, independentemente de qualquer ciclo hormonal ou do estado fisiológico da mulher, sendo que apenas $0,01 \%$ ovulam, e os demais degeneram. A depleção dos folículos ovarianos ocorre independentemente de fatores fisiológicos e ambientais até a fase de perimenopausa. A fase da perda folicular acelerada e sua velocidade irão determinar a idade de ocorrência da menopausa. Parece ser a data da menopausa geneticamente "programada" para cada mulher, mas esta pode ser influenciada por alguns fatores, como a paridade, a nutrição, a raça e o tabagismo, em até três anos (Ginsburg, 1991).

Alguns autores observaram que a paridade está ligada à idade à menopausa: mulheres nulíparas têm menopausa mais precocemente, enquanto que o aumento da paridade correlaciona-se a uma menopausa mais tardia (Kato et al., 1998). Outros, entretanto, não evidenciaram essa associação (McKinlay et al., 1985).

Esse fato sugere que condições que causam longos períodos de anovulação durante a vida reprodutiva, como a paridade (Parazzini et al., 1992), uso de contraceptivos orais e padrão menstrual irregular ou mesmo a menarca precoce, podem estar associados a um atraso na menopausa. Isso é interpretado de acordo com o conceito da exaustão dos folículos disponíveis ser a causa da menopausa, pois o fator mais importante para determinar a idade de ocorrência da menopausa é o número de folículos ovarianos.

A obesidade é relacionada a um excesso de produção de estrógenos endógenos, tanto na pré como na pós-menopausa, e tem sido associada a um padrão menstrual aberrante e anovulatório. Sherman et al. (1981) evidenciaram que mulheres que foram obesas até os 18 anos de idade tinham uma menopausa mais tardia do que as que só desenvolveram obesidade mais tardiamente durante a vida reprodutiva. Outros (Kato et al., 1998), no entanto, mostraram uma associação entre a obesidade e a idade à menopausa, quando o índice de massa corpórea foi medido na perimenopausa. Esses dados sugerem que a obesidade poderia maximizar o período da vida reprodutiva, como foi observa- 
do neste estudo, porém sem significância estatística.

Além de um período de vida reprodutiva mais prolongado e um total de ciclos menstruais em maior número, o ambiente estrogênico das mulheres obesas pode ser mais intenso que das magras, devido a uma maior conversão da androstenediona em estrona e uma maior concentração de estrógeno livre, pela diminuição do nível da globulina carreadora de hormônios sexuais, que geralmente acompanha a obesidade.

Neste estudo não se observou associação entre o tabagismo e a idade de ocorrência da menopausa, diferentemente de estudos prévios que indicam uma idade à menopausa de um a dois anos mais precoce em fumantes, quando comparadas às não-fumantes (Bromberger et al., 1997). Análise mais refinada da média etária à menopausa segundo o tempo de tabagismo e o número de cigarros ao dia, também não demonstrou qualquer diferença estatisticamente significativa. Razões para essa discrepância não são claras, mas vale a pena ressaltar que estudos de base populacional, ou seja, de população não-hospitalar, geralmente encontram menos efeito do tabagismo sobre a idade à menopausa, quando comparados a estudos que incluem sujeitos doentes.

A consistência dos dados deste estudo minimiza, mas não exclui, a possibilidade de alguns viéses em potencial, incluindo o fato de ser um estudo de coorte transversal e retrospectivo em relação à idade à menopausa. $\mathrm{O}$ viés de recordação é possível tanto para a idade à menopausa, como também para várias medidas dos fatores de exposição anteriores à menopausa.

Segundo Colditz et al. (1987), a reprodutibilidade para a idade à menopausa pelas mulheres, após o evento, é geralmente boa para as de meia-idade, nas quais um tempo relativamente curto se passou desde a menopausa. Esses autores observaram que a reprodutibilidade da idade de ocorrência da menopausa natural variou em aproximadamente um ano para $82 \%$ das mulheres, e para aquelas com menopausa cirúrgica a reprodutibilidade foi de $95 \%$. Portanto, concluiu-se que os dados referentes a detalhes sobre o estado menopausal podem ser relatados com alto grau de reprodutibilidade e acurácia nos anos imediatamente seguintes à menopausa, porém são mais confiáveis quando coletados prospectivamente em estudos longitudinais.

Em estudo realizado por Hahn et al. (1997), onde também se estudou a confiabilidade sobre idade à menopausa relatada pela própria mulher, evidenciou-se que mulheres com menopausa cirúrgica relataram a idade à meno-
Tabela 4

Idade mediana à menopausa segundo características reprodutivas e índice de massa corpórea $(n=455)$.

\begin{tabular}{|c|c|c|c|}
\hline Variável & Idade mediana & $\mathrm{n}$ & $p^{*}$ \\
\hline Idade na menarca (anos) & & & 0,509 \\
\hline Até 11 & 52,2 & 101 & \\
\hline $12-14$ & 51,2 & 284 & \\
\hline$>14$ & 50,8 & 70 & \\
\hline Paridade & & & 0,157 \\
\hline Até 3 & 50,9 & 242 & \\
\hline$\geq 3$ & 51,5 & 213 & \\
\hline Número de abortos & & & 0,027 \\
\hline 0 & 51,7 & 301 & \\
\hline$\geq 1$ & 50,7 & 154 & \\
\hline $\begin{array}{l}\text { Antecedente de uso de método } \\
\text { anticoncepcional hormonal }\end{array}$ & & & 0,018 \\
\hline Não & 50,6 & 164 & \\
\hline $\operatorname{Sim}$ & 51,6 & 291 & \\
\hline $\begin{array}{l}\text { Antecedente de uso de método } \\
\text { anticoncepcional hormonal }\end{array}$ & & & 0,056 \\
\hline Não & 50,9 & 194 & \\
\hline $\operatorname{Sim}$ & 51,5 & 259 & \\
\hline Antecedente de laqueadura & & & 0,224 \\
\hline Não & 51,1 & 196 & \\
\hline $\operatorname{Sim}$ & 51,3 & 259 & \\
\hline Índice de massa corporal $\left(\mathrm{kg} / \mathrm{m}^{2}\right)$ & & & 0,291 \\
\hline$\leq 30,00$ & 50,9 & 352 & \\
\hline$>30,00$ & 52,0 & 103 & \\
\hline
\end{tabular}

* Teste de Wilcoxon generalizado (Gehan).

Tabela 5

Variáveis associadas à taxa acumulada das mulheres à menopausa natural - Regressão de $\operatorname{Cox}^{\star}(n=448)^{\star \star}$.

\begin{tabular}{lccc}
\hline Variável $^{\star \star \star}$ & Coef. & E.P.Coef. & P \\
\hline Sem variáveis associadas & - & - & - \\
\hline
\end{tabular}

* Análise de sobrevivência.

** Sete mulheres com variáveis ignoradas.

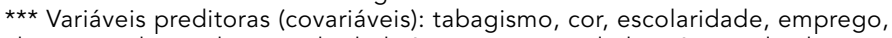
classe social, estado marital, idade à menarca, paridade, número de abortamento, antecedente de uso de método anticoncepcional hormonal, não-hormonal, laqueadura e índice de massa corpórea. 
pausa com mais confiabilidade do que as com menopausa natural, e que a confiabilidade diminui com o tempo desde a ocorrência da menopausa, com o avançar da idade e quanto menor for o nível educacional.

A validade e a reprodutibilidade do auto-relato da idade à menopausa em mulheres holandesas mostraram diferença de até um ano em $70 \%$ dos casos. Nas mulheres com menopausa cirúrgica, esta taxa foi de $80 \%$. A validade dos dados diminui com o passar dos anos desde a menopausa. Após sete a nove anos da ocorrência da menopausa, as mulheres tendem a subestimar sua idade à menopausa em aproximadamente 0,3 anos, independentemente de sua idade cronológica. Como conseqüência, em estudos que relacionam a idade à menopausa com a ocorrência de doenças ou mortalida- de, este risco estimado pode ser sub ou superestimado (Den Tonkelaar, 1997).

Apesar deste estudo ser realizado de forma retrospectiva em relação à idade de ocorrência da menopausa, este dado pode ser considerado de confiabilidade, pois o tempo médio decorrido desde a menopausa foi de 7,2 anos, o que daria uma reprodutibilidade entre $80 \%$ a $95 \%$, de acordo com os estudos citados anteriormente.

Os resultados desta pesquisa, a primeira de base populacional com mulheres climatéricas brasileiras, ajudarão não só o planejamento de serviços e rotinas de assistência, como também o delineamento do ensino nas instituições pertinentes e, sobretudo, servirão de base para a necessidade de pesquisas fundamentadas em uma realidade brasileira.

\section{Agradecimentos}

Este trabalho recebeu incentivo da Fundação de Amparo à Pesquisa do Estado de São Paulo (FAPESP), do Fundo de Apoio ao Ensino e Pesquisa (FAEP) da Faculdade de Ciências Médicas da Universidade Estadual de Campinas e do Conselho Nacional de Desenvolvimento Científico e Tecnológico (CNPq). 


\section{Referências}

ALMEIDA, P. M. \& WICKERHAUSER, H., 1991. O Critério ABA/ABIPEME - Em Busca de uma Atualização. São Paulo: Associação Brasileira dos Institutos de Pesquisa de Mercado.

BENJAMIN, F., 1960. The age of the menarche and of the menopause in white Southern African women and certain factors influencing these times. Southern African Medical Journal, 3:316-320.

BOULET, M. J.; ODDENS, B. J.; LEHERT, P.; VEMER, H. M. \&VISSER, A., 1994. Climacteric and menopause in seven Southeast Asian countries. Maturitas, 19: 157-176.

BRAND, P. C. \& LEHERT, P. H., 1978. A new way of looking at environmental variables that may affect the age at menopause. Maturitas, 1:121-132.

BROMBERGER, J. T.; MATTHEWS, K. A.; KULLER, L. H.; WING, R. R.; MEILAHN, E. N. \& PLANTINGA, P., 1997. Prospective study of the determinants of age at menopause. American Journal of Epidemiology, 145:124-133.

CHOMPOOTWEEP, S.; TANKEYOON, M.; YAMARAT, K.; POOMSUWAN, P. \& DUSITSIN, N., 1993. The menopausal age and climacteric complaints in Thai women in Bangkok. Maturitas, 17:63-71.

COLDITZ, G. A.; STAMPFER, M. J.; WILLETT, W. C.; STASON, W. B.; ROSNER, B.; HENNEKENS, C. H. \& SPLIZER, F. E., 1987. Reproducibility and validity of self-reported menopausal status in a perspective cohort study. American Journal of Epidemiology, 126:319-325.

DEN TONKELAAR, I., 1997. Validity and reproducibility of self-reported age at menopause in women participating in the DOM-project. Maturitas, 27: 117-123.

FONSECA, A. M.; HEGG, R.; GUARNIERI-NETTO, C. D.; MELO, N. R.; FILASSI, J. R. \& SALVATORE, C. A., 1985. Climatério: Aspectos epidemiológicos e clínicos. Revista Brasileira de Terapêutica, 14:34533489.

GINSBURG, J., 1991. What determines the age of menopause? BMJ, 302:1288-1289.

HAHN, R. A.; EAKER, E. \& ROLKA, H., 1997. Reliability of reported age at menopause. American Journal of Epidemiology, 146:771-775.

HALBE, H. W.; FONSECA, A. M.; ASSIS, J. S.; VITÓRIA, S. M.; ARIE, M. H. A.; ELIAS, D. S.; MELO, N. R. \& BAGNOLI, V. R., 1990. Aspectos epidemiológicos e clínicos em 1.319 pacientes climatéricas. Revista de Ginecologia e Obstetrícia, 1:182-194.
KATO, I.; TONIOLO, P.; AKHNEDKHANOV, A.; KOENIG, K. L.; SHORE, R. \& ZELENIUCH-JACQUOTTE, A., 1998. Prospective study of factors influencing the onset of natural menopause. American Journal of Epidemiology, 51:1271-1276.

KISH, L., 1972. Muestreo de conglomerado y submuestreo. In: Muestreo de Encuestas (L. Kish, org.), pp. 182-219, México, DF: Editorial Trillas.

KWAWUKUME, E. Y.; GHOSH, T. S. \& WILSON, J. B., 1993. Menopausal age of Ghanaian women. International Journal of Gynaecology and Obstetrics, 40:151-155.

McKINLAY, S.; BIFANO, N. L. \& McKINLAY, J. B., 1985. Smoking and age at menopause in women. Annals of Internal Medicine, 103:350-356.

McKINLAY, S.; JEFFERYS, M. \& THOMPSON, B., 1972. An investigation of the age at menopause. Journal of Biosocial Science, 4:161-173.

PARAZZINI, F.; NEGRI, E. \& LA VECCHIA, C., 1992. Reproductive and general lifestyle determinants of age at menopause. Maturitas, 15:141-149.

PINTO NETO, A. M.; COSTA-PAIVA, L. H. S.; MIRANDA, W. A.; ZABAGLIA, S. F. C.; NASCIMENTO, F. L. B.; CAIRO, A. A. A.; PETTA, C. A. \& LANE, E., 1992. Patologia mamária na mulher climatérica. Jornal Brasileiro de Ginecologia, 102:309-312.

RIZK, D. E. E.; BENER, A.; EZIMOKHAI, M.; HASSAN, M. Y. \& MICALLEF, R., 1998. The age and symptomathology of natural menopause among United Arab Emirates women. Maturitas, 29:197-202.

SHERMAN, B.; WALLACE, R.; BEAN, J. \& SCHALABAUGH, L., 1981. Relation of body weight to menarcheal and menopausal age: Implications for breast cancer risk. Journal of Clinical Endocrinology and Metabolism, 52:488-493.

UTIAN, W. H. \& SCHIFF, I., 1994. Gallup survey on women's knowledge, information sources, and attitudes to menopause and hormone replacement therapy. Menopause, 1:39-48.

WEHBA, S.; FERNANDES, C. E.; MELO, N. R.; FERREIRA, J. A. S.; FARIA Jr., D. \& ROUCOURT, S., 1998. Aspectos epidemiológicos, clínicos e de diagnóstico do Climatério. In: Hormonioterapia e Psicofarmacoterapia na Saúde da Mulher (C. E. Fernandes, N. R. Melo, C. N. Soares \& S. Wehba, org.), pp. 15-22, São Paulo: Sociedade Brasileira de Climatério.

Recebido em 4 de agosto de 2000

Versão final reapresentada em 12 de abril de 2002

Aprovado em 28 de junho de 2002 